\title{
Review of: "Coexistence of Negative and Positive Photoconductivity in Few-Layer PtSe 2 Field-Effect Transistors"
}

\author{
Nadia Martucciello ${ }^{1}$ \\ 1 Italian National Research Council
}

Potential competing interests: The author(s) declared that no potential competing interests exist.

The paper is of a great interest.

This is a study on p-type conduction in back-gated field effect transistors with a channel made of ultrathin PtSe2 film. Authors showed that positive and negative photoconductivity can occur simultaneously and that the dominance of one over the other depends on the environmental pressure.

The experiments are very well prepared and described in the paper. The discussion of results is convincing. I recommend to read it! 